\title{
Electric Vehicle Charging Station Placement
}

\author{
Albert Y.S. Lam, Yiu-Wing Leung, and Xiaowen Chu \\ Department of Computer Science \\ Hong Kong Baptist University \\ Hong Kong \\ Email: albertlam@ieee.org
}

\begin{abstract}
Transportation electrification is one of the essential components in the future smart city planning and electric vehicles (EVs) will be integrated into the transportation system seamlessly. Charging stations are the main source of energy for EVs and their locations are critical to the accessibility of EVs in a city. They should be carefully situated so that an EV can access a charging station within its driving range and cruise around anywhere in the city upon being recharged. In this paper, we formulate the Electric Vehicle Charging Station Placement Problem, in which we minimize the total construction cost subject to the constraints for the charging station coverage and the convenience of the drivers for EV charging. We study the properties of the problem, especially its NP-hardness, and propose an efficient greedy algorithm to tackle the problem. We perform a series of simulation whose results show that the greedy algorithm can result in solutions comparable to the mixed-integer programming approach and its computation time is much shorter.
\end{abstract}

\section{INTRODUCTION}

Modern civilization relies heavily on fossil fuels to support construction, military protection, and people's mobility. Due to the world's shortage of fossil fuels, nations compete to secure enough reserves of natural resources for sustainability. Seeking alternative energy sources becomes crucial to a nation's future development. One of the major sources of fossil fuel consumption is transportation. Most of our daily heavily demanded transportation, including buses and private cars, is powered by gasoline. A major consequence of burning fossil fuels is the release of tremendous amount of harmful gases, which partially constitutes the global warming effect and deteriorates people's health. Electricity is considered as the most universal form of energy, which can be transformed from and to another form effectively. By converting the endurable renewable energy, like solar and wind, to electricity, we can manipulate energy in a much cleaner manner. Electrification of transportation like deployment of electric vehicles (EVs) can not only alleviate our demand on fossil fuels, but also foster a better environment for living. Therefore, EVs will become the major components in the future transportation system.

Incorporating EVs into an existing self-contained transportation system is challenging. Solely expanding the population of EVs in a city without enough road connections and corresponding charging and parking infrastructure will suppress the practicability of EVs due to their limiting moving ranges. Conversely, constructing the facilities with low utilization will result in a waste of resources. Moreover, existing gas stations are primarily designed for gas refueling; combining charging infrastructure with the conventional gas stations may not be appropriate as the relatively longer charging process will saturate the limited space of the gas stations. We need to carefully plan EV charging facilities to modernize our cities. To be precise, we study how EVs will be integrated into the transportation system seamlessly and this will help make our cities "smart". To do this, we study the Electric Vehicle Charging Station Placement Problem (EVCSPP) by finding the best locations to construct charging stations in a city.

Technology advances rapidly while the smart city plan should take a much longer timespan. The plan should cater for the residents. Hence we focus on the long-term human aspects rather than the technological ones. An EV can always access a charging station within its capacity anywhere in the city. Charging stations should be built widely enough such that the moving range of an EV can be extended to every corner of the city by having the EV recharged at a charging station available nearby. We study where charging stations should be constructed in a city such that we can minimize the construction cost with coverage extended to the whole city and fulfillment of drivers' convenience. In this paper, we formulate the problem as an optimization, based on the charging station accessibility and coverage in the city. We also propose methods to solve the problem.

EVs take the central role in this paper and they have been being studied since the boom of the smart grid. Most of the existing work on EVs is related to studying the relationship between EVs and the grid, i.e., how power is transferred from and to the grid. Besides scheduling charging [1], [?], in vehicle-to-grid (V2G) systems, hundreds of EVs coordinate to act as a power source selling power back to the grid or to support auxiliary services like regulation. A multi-layer market for $\mathrm{V} 2 \mathrm{G}$ energy trading was proposed in [2]. The market price was settled via double auction and the mechanism could maximize the EVs' revenues. In [3], a queueing network was utilized to model the dynamics of EVs participating in V2G. The model could facilitate service contract engagement for regulation ancillary services. [4] investigated the joint scheduling of EV and unit commitment and this allowed us to optimize the system's total running cost with the presence of EVs. [5] discussed the incorporation of photovoltaic (PV) equipment into charging stations. It considered that charging facilities equipped with PV panels and the stored solar energy, together with the power requested from the grid, can be used to power EVs. [6] and [7] studied the impact of EV charging to the performance of power distribution networks with the presence of charging stations, which can represent rapid heavy loads. [6] illustrated the effect of fast-charging EVs in terms of power-flow, short-circuit and protection while [7] proposed a new smart load management strategy to coordinate EVs for peak load shaving, power loss minimization, and voltage profile improvement. However, in this paper, we no longer 
focus on a standalone system but enlarge the scope to the city level, with particular interest in charging stations. We concern more about how EVs influence the growth of a smart city.

[8] investigated the location and sizing issues of EV charging station planning. It considered environmental factors and service radius of EV charging stations when selecting candidate sites, but the focus was on determining the charging station sizing in terms of power system factors, e.g., transformer capacity, reaction power, voltage, current, etc. The site selection method was over-simplified. [9] considered the location and sizing problem of charging station placement with traffic flow by minimizing the annual integrated and operating cost of charging stations, and charging cost. Particle swarm optimization heuristic was adopted to compute the solution of the non-convex problem. [10] discussed the design of power architectures and power electronics circuit topologies for high power superfast EV charging stations with enhanced grid support functionality. However, none of them examines the placement issue in terms of EVs' reachability and accessibility for city planning.

The rest of this paper is organized as follows. We give the necessary backgrounds to define the problem in Section II and formulate the problem and discuss its complexity in Section III. Section IV presents two solution methods, one of which is our greedy algorithm. In Section V, simulation results are provided for performance evaluation and we conclude this paper in VI.

\section{BACKGROUNDS}

In this section, we give the system model and the basic requirements for defining EVCSPP.

We model a city with an undirected graph $G=(\mathcal{N}, \mathcal{E})$, where $\mathcal{N}$ and $\mathcal{E}$ denote the sets of possible sites for constructing charging stations and connections between pairs of sites, respectively. Suppose $|\mathcal{N}|=n$. Let $d: \mathcal{N} \times \mathcal{N} \rightarrow \mathbb{R}^{+}$be the distance function, where $d(i, j)$ denotes the distance of the shortest path from nodes $i$ to $j$ by traversing the connections. ${ }^{1}$ Let $f_{i}$ be the capacity of node $i$ representing the average capacity of charging service supported if a charging station is constructed at location $i$. It is related to the size of the site and traffic conditions around. Each node $i$ also has a demand requirement $F_{i}$, which refers to its average local charging demand. The more EVs are based at location $i$, the higher $F_{i}$ is. The values of $F_{i}$ can be estimated from the population density and the EV penetration rate.

We define $D$ to be the average distance able to be traversed by most typical EV models available in the market when being fully charged. A subset of nodes $\mathcal{N}^{\prime} \subset \mathcal{N}$ is said to be reachable by $D$ if the following conditions hold:

C1) For each $i \in \mathcal{N}^{\prime}$, there exists a node $j \in \mathcal{N}^{\prime}$ such that $d(i, j) \leq D$

C2) For each $i \in \mathcal{N}$, the total capacity, constituted from those nodes $j \in \mathcal{N}^{\prime}$ such that $d(i, j) \leq \alpha D$ with discount factor $\alpha \in(0,1]$, is greater than or equal to $F_{i}$; and

\footnotetext{
${ }^{1}$ The distance $d(i, j)$ refers to the distance of an actual path connecting locations $i$ and $j$ but not the Euclidean distance.
}

C3) For any $i, j \in \mathcal{N}^{\prime}$, suppose $h_{i j}$ be the number of hops of the shortest path from $i$ to $j$ in $G$. The distance of the path $d(i, j)$ should be smaller than or equal to $h_{i j} D$.

$\mathcal{N}^{\prime}$ represents the set of locations which have been selected to have charging stations constructed. A city is well planned if $\mathcal{N}^{\prime}$ is reachable by $D$. With condition $\mathrm{C} 1$, an $\mathrm{EV}$, which has been fully charged at a location, can recharge again at another site within distance $D$ away. $\mathrm{C} 1$ guarantees that EVs will not be confined in one single location (or area). Condition C2 says that the local charging demand at a location (e.g., $F_{i}$ at node i) must be satisfied by the total charging capacities contributed by those charging stations located within distance $\alpha D$ away. $\alpha$ is used to model the tolerance of drivers to move away from its location for charging. Its maximum value is one because an EV can traverse for a distance at most $D$. The smaller $\alpha$, the more conservative the model is, i.e., more charging stations should be placed around every possible location. With condition C3, the charging station network, where each charging station is separated with another of at most distance $D$, spans the whole city. To summarize, the conditions all together guarantee that the serving areas of the charging stations cover every corner of the city.

\section{PROBLEM DEFINITION}

In this section, we formulate EVCSPP based on the system model and the requirements defined in the previous section. We also prove the problem NP-hard.

\section{A. Formulation}

Let $x_{i}$ be the decision (boolean) variable indicating if node $i$ is chosen for placement and $c_{i}$ be its construction cost. We minimize the total cost as the objective, i.e., $\sum_{i=1}^{n} c_{i} x_{i}$.

For each $i$, we define $\mathcal{N}_{i}^{\alpha D}=\{j \in \mathcal{N} \mid d(i, j) \leq \alpha D\}$, representing the set of nodes (including node $i$ itself) within distance $\alpha D$ from $i$. We can re-state condition $\mathrm{C} 2$ as

$$
\sum_{j \in \mathcal{N}_{i}^{\alpha D}} f_{j} x_{j} \geq F_{i}, \forall i \in \mathcal{N} .
$$

As condition C3 holds for any pair of nodes, C3 implies C1. To re-state $\mathrm{C} 3$, we first create a graph $\hat{G}=(\hat{\mathcal{N}}, \hat{\mathcal{E}})$, where $\hat{\mathcal{N}}$ is set to $\mathcal{N}$ and $\hat{\mathcal{E}}$ is equal to $\{(i, j) \mid i, j \in \mathcal{N}, d(i, j) \leq D, i \neq j\}$. Consider the example of $G$, composed of 8 nodes, given in Fig. 1(a), where the number on a connection indicates the distance between the nodes on the two ends. With $D=6$, we have the corresponding $\hat{G}$ in Fig. 1(b) (ignore node $0^{1}$, which will be explained later). Consider those nodes $i$ in $G$ with $x_{i}=1$ (i.e., $\mathcal{N}^{\prime}$ ) and they constitute the corresponding induced subgraph $H$ of $\hat{G}$. Condition $\mathrm{C} 3$ is equivalent to having $H$ connected. In other words, $H$ has one single connected component. Instead of inspecting the original graph $G$, we can focus on $\hat{G}$ to define the problem. Similar to [11], we adopt a network flow model to address C3. Imagine that there is a source node $0^{i}$ attached to node $i$ and it has $n$ units of flow available to be sent along $\hat{G}$ through node $i$. Let $0 \leq x_{0}^{i} \leq n$ be the residue of flow not consumed by the network. Each node $j$ with $x_{j}=1$ will consume one unit of flow. For each edge $(j, k) \in \hat{\mathcal{E}}$, we indicate the amount of flow on $(j, k)$ originated from $0^{i}$ with variable $y_{j k}^{i}$. Hence, we can guarantee those nodes $j$ with 
$x_{j}=1$ being reached from node $i$ on $\hat{G}$ with the following constraints:

$$
\begin{aligned}
x_{0}^{i}+y_{0 i}^{i} & =n, \\
0 \leq y_{j k}^{i} & \leq n x_{k}, \forall(j, k) \in \hat{\mathcal{E}} \cup\left(0^{i}, i\right), \\
\sum_{j \mid(j, k) \in \hat{\mathcal{E}}} y_{j k}^{i} & =x_{k}+\sum_{l \mid(k, l) \in \hat{\mathcal{E}}} y_{k l}^{i}, \forall k \in \hat{\mathcal{N}} \\
\sum_{j \in \hat{\mathcal{N}}} x_{j} & =y_{0 i}^{i}, \\
0 & \leq x_{0}^{i} .
\end{aligned}
$$

Eq. (1) says that the total amount of flow going out of $y_{0 i}^{i}$ and retained $x_{0}^{i}$ in the source $0^{i}$ is $n$. Eq. (2) confines that only a sink can receive incoming flow. Eq. (3) describes that the total incoming flow to a node is equal to the total outgoing flow plus the amount for a sink. Eq. (4) explains that the total flow getting out of the source is equal to the total absorbed by the sinks and (5) restricts non-negative residue remained in the source.

Note that (1)-(5) require node $i$ to be selected for charging station construction. Otherwise, no flow from the Source $0^{i}$ is allowed to be delivered to the sinks. To cater for this requirement, we attach a source node to each node in $\hat{\mathcal{N}}$ and the overall mathematical formulation of EVCSPP is modified accordingly as follows:

$$
\begin{array}{ll}
\text { minimize } & \sum_{i=1}^{n} c_{i} x_{i} \\
\text { subject to } & \sum_{j \in \mathcal{N}_{i}^{\alpha D}} f_{j} x_{j} \geq F_{i}, \forall i \\
& x_{i}=\{0,1\}, \forall i \\
& x_{0}^{i}+y_{0 i}^{i}=n, \forall i \in \hat{\mathcal{N}} \\
& 0 \leq y_{j k}^{i} \leq n x_{i} x_{k}, \forall(j, k) \in \hat{\mathcal{E}} \cup\left(0^{i}, i\right), \forall i \in \hat{\mathcal{\mathcal { N }}} \\
& \sum_{j \mid(j, k) \in \hat{\mathcal{E}}} y_{j k}^{i}=x_{i} x_{k}+\sum_{l \mid(k, l) \in \hat{\mathcal{E}}} y_{k l}^{i}, \forall i, k \in \hat{\mathcal{N}} \\
& x_{i} \sum_{j \in \hat{\mathcal{N}}} x_{j}=y_{0 i}^{i}, \forall i \in \hat{\mathcal{N}} \\
& 0 \leq x_{0}^{i}, \forall i \in \hat{\mathcal{N}} .
\end{array}
$$

\section{B. Complexity}

The decision version of the problem can be framed as follows:

Let $N(H)$ be the set of nodes associated to the induced subgraph $H$. Each node $i$ has a capacity $f_{i} \in \mathbb{Z}^{+}$and a demand $F_{i}$ and is associated with the node set $\mathcal{N}_{i}^{\alpha D}$. Given an undirected graph $\hat{G}=(\hat{\mathcal{N}}, \hat{\mathcal{E}})$, with node cost $c_{i} \in \mathbb{Z}^{+}, \forall i$, a cost bound $C \in \mathbb{Z}^{+}$, does there exist an induced subgraph $H$ of $\hat{G}$ such that

1) For each $i \in \hat{\mathcal{N}}, \sum_{j \in \mathcal{N}_{i}^{\alpha D} \cap N(H)} f_{j} \geq F_{i}$,

2) $H$ is connected, and

3) $\sum_{i \in N(H)} c_{i} \leq C$ ?

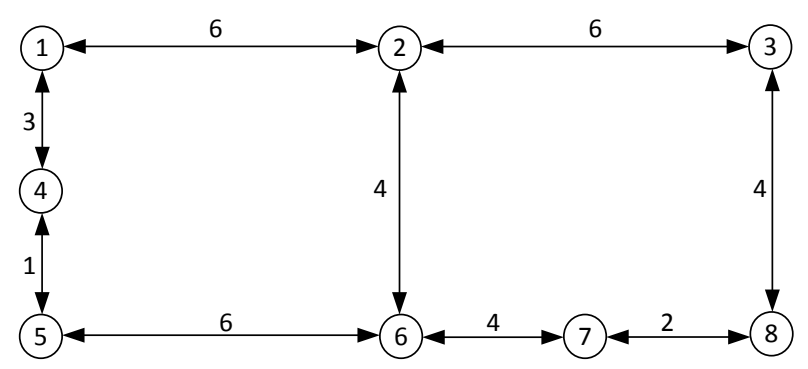

(a) $G$

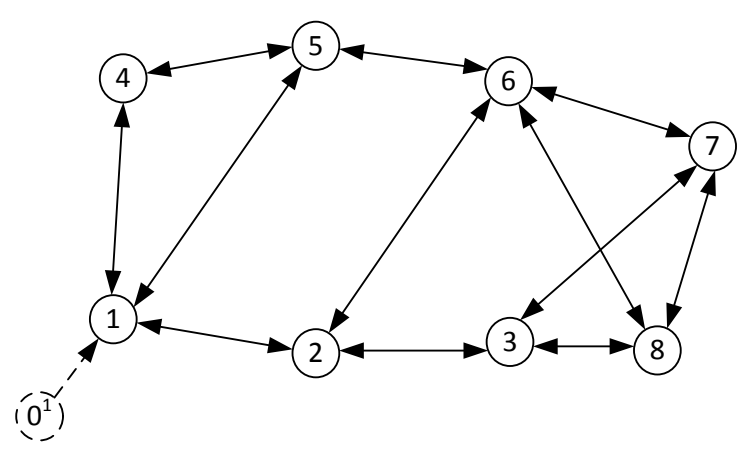

(b) $\hat{G}$

Fig. 1. An example of 8 nodes.

Theorem 1: The decision version of EVCSPP is NPcomplete.

Proof: Similar to [11], we construct a reduction from the vertex cover problem (VCP) to EVCSPP. In graph $\tilde{G}=$ $(\tilde{\mathcal{N}}, \tilde{\mathcal{E}})$, a vertex cover is a subset of nodes $\mathcal{N}^{\prime} \subset \tilde{\mathcal{N}}$ such that each edge $(i, j) \in \tilde{\mathcal{E}}$ has $i, j$, or both in $\tilde{\mathcal{N}}$. Without loss of generality, we assume $\tilde{\mathcal{E}} \neq \emptyset$. VCP determines if there exists a vertex cover $\mathcal{N}^{\prime}$ of $\tilde{G}$ with $\left|\mathcal{N}^{\prime}\right| \leq C$.

We create a graph $\hat{G}=(\hat{\mathcal{N}}, \hat{\mathcal{E}})$, where $\hat{\mathcal{N}}=\tilde{\mathcal{N}} \cup \tilde{\mathcal{E}}$ and $\hat{\mathcal{E}}$ is constructed as follows. For each pair of distinct nodes $i, j \in \tilde{\mathcal{N}}$, we create an edge $(i, j)$ in $\hat{\mathcal{E}}$; for each $e=(i, j) \in \tilde{\mathcal{E}}$, we append $(i, e)$ and $(e, j)$ to $\mathcal{\mathcal { E }}$. For each $i \in \tilde{\mathcal{N}}$, its cost is set as $c_{i}=1$ and zero otherwise. For each $e \in \tilde{\mathcal{E}}$, we set $f_{e}=1$ and zero otherwise. We also set $\mathcal{N}_{i}^{\alpha D}=\tilde{\mathcal{E}}$ and $F_{i}=|\tilde{\mathcal{E}}|$ for all $i \in \hat{\mathcal{N}}$.

We claim that CVP on $\tilde{G}$ with cost upper bound $C$ if and only if EVCSPP has a solution with cost at most $C$.

Let $\mathcal{N}^{\prime}$ be a vertex cover of $\tilde{G}$ with $\left|\mathcal{N}^{\prime}\right| \leq C$ and $H$ be the induced subgraph of $\hat{G}$ by nodes $\mathcal{N}^{\prime} \cup \tilde{\mathcal{E}}$. It is easy to verify that $\left|\mathcal{N}_{i}^{\alpha D} \cap N(H)\right|=|\tilde{\mathcal{E}}|$ and thus $\sum_{j \in \mathcal{N}_{i}^{\alpha D} \cap N(H)} f_{j}=|\tilde{\mathcal{E}}|=F_{i}$. As $\mathcal{N}^{\prime}$ is a vertex cover, each $e=(i, j) \in \tilde{\mathcal{E}}$ must have at least one of $i$ and $j$ in $\tilde{\mathcal{N}}$ and thus $H$ must contain an edge $(e, k)$ for some $k \in \mathcal{N}^{\prime}$. Moreover, $\tilde{\mathcal{N}}$ forms a clique in $\hat{G}$. Hence, $H$ must be connected. Since each $e \in \tilde{\mathcal{E}} \subset \hat{\mathcal{N}}$ imposes no cost, $H$ has the same cost as $\mathcal{N}^{\prime}$ in $\tilde{G}$. Therefore, EVCSPP has a solution with cost at most $C$.

Consider that an induced subgraph $H$ is a solution of EVCSPP. We set $\mathcal{N}^{\prime}=N(H) \cap \tilde{\mathcal{N}}$. $H$ contains $\tilde{\mathcal{E}}$ : As $f_{j}=1$ for $j \in \tilde{\mathcal{E}}$, for any $i \in \hat{\mathcal{N}}, F_{i}=|\tilde{\mathcal{E}}|$ guarantees $\tilde{\mathcal{E}} \subset N(H)$. 
Since $H$ is connected, each $i \in \mathcal{N}^{\prime}$ must have an edge with an $e \in \tilde{\mathcal{E}}$ in $\hat{G}$. Moreover, $\mathcal{N}^{\prime}$ has at most $C$ nodes. Hence, $\mathcal{N}^{\prime}$ is a vertex cover of $\tilde{G}$ with $\left|\mathcal{N}^{\prime}\right| \leq C$.

\section{Corollary 1: EVCSPP is NP-hard.}

\section{PRoposed Solutions}

Since there are quadratic terms in some of the equality constraints, problem (6) is neither a mixed-integer linear program (MILP) nor a mixed-integer quadratic program, and thus solvers for standard mixed-integer programming (MIP) may not be applied. However, we will show that the problem can be transformed to a number of MILP's. We will also discuss a greedy algorithm which even works for the original formulation (6).

\section{A. Mixed-Integer Linear Programs}

Eqs. (1)-(5) can be used to guarantee that the solution subgraph constituted by all nodes $j$ with $x_{j}=1$ is connected as long as $x_{i}=1$. If we assume that node $i$ will be one of locations for charging station construction, i.e., $x_{i}=1$, problem (6) becomes

$$
\begin{aligned}
\text { minimize } & \sum_{i=1}^{n} c_{i} x_{i} \\
\text { subject to } & \sum_{j \in \mathcal{N}_{i}^{\alpha D}} f_{j} x_{j} \geq F_{i}, \forall i, \\
& x_{i}=\{0,1\}, \forall i \\
& x_{0}^{i}+y_{0 i}^{i}=n, \\
& 0 \leq y_{j k}^{i} \leq n x_{k}, \forall(j, k) \in \hat{\mathcal{E}} \cup\left(0^{i}, i\right), \\
& \sum_{j \mid(j, k) \in \hat{\mathcal{E}}} y_{j k}^{i}=x_{k}+\sum_{l \mid(k, l) \in \hat{\mathcal{E}}} y_{k l}^{i}, \forall k \in \hat{\mathcal{N}} \\
& \sum_{j \in \hat{\mathcal{N}}} x_{j}=y_{0 i}^{i}, \\
& 0 \leq x_{0}^{i} .
\end{aligned}
$$

Problem (7) is an MILP and it can be solved with standard MIP solvers applying methods like branch-and-bound. Now the question becomes which node $i$ should be chosen for this purpose. As there is no trivial way to choose such a node $i$, we need to apply (7) to every possible node. In other words, we solve (7) $n$ times, each of which has $i$ set to one of $\{1, \ldots n\}$. The solution of (6) is the best feasible one of the MILPs' solutions. ${ }^{2}$ However, they are usually subject to long computation time and only applicable to small problem instances.

\section{B. Greedy Algorithm}

Here we provide another method to tackle the problem. We present an efficient greedy algorithm and requires much shorter computation time. Before discussing its details, we have the following lemma to facilitate its development.

\footnotetext{
${ }^{2}$ The MILPs for some fixed nodes may result in infeasible solutions to the original problem. The best solution is selected among the feasible ones.
}

Lemma 1: Problem (6) is feasible if and only if $x=$ $\left[x_{1}, \ldots, x_{n}\right]=[1, \ldots, 1]$ is a feasible solution, which gives an upper bound with the objective function values $\sum_{i=1}^{n} c_{i}$.

Proof: First we consider the only if-direction. As the problem is feasible, there exists a feasible $x^{\prime}=\left[x_{1}^{\prime}, \ldots, x_{n}^{\prime}\right]$, composed of some 0's and/or 1's, satisfying constraints (6b)(6h). If $x_{i}^{\prime}=1$ for all $i$, then we have the result. Consider that there is at least one $j$ such that $x_{j}^{\prime}=0$. If we produce another $x^{\prime \prime}$ by modifying $x_{j}^{\prime}$ with value one, besides (6c), $x^{\prime \prime}$ will always satisfy constraint (6b), as we will stay unchanged or increase the sum on the left-hand side of (6b). Moreover, as $0<\alpha \leq 1$, if $x_{j}^{\prime}=0$ satisfies (6b), there exists at least one node $k$ with $x_{k}^{\prime}=x_{k}^{\prime \prime}=1$ within distance $D$ away from node $j$. In this way, if we have $x_{j}^{\prime \prime}=1$, we will attach node $j$ to the subgraph induced by $x^{\prime}$ through node $k$. In other words, the subgraph induced by $x^{\prime \prime}$ is still connected, i.e., satisfying (6d)-(6h). We can repeat this process until we change all 0's to 1 's and this produces $x$ with upper bound $\sum_{i=1}^{n} c_{i}$.

The if-direction is trivial. We complete the proof.

Corollary 2: If $x=\left[x_{1}, \ldots, x_{n}\right]=[1, \ldots, 1]$ is not feasible, EVCSPP is infeasible.

Corollary 2 can be used as a test to check the feasibility of a problem instance.

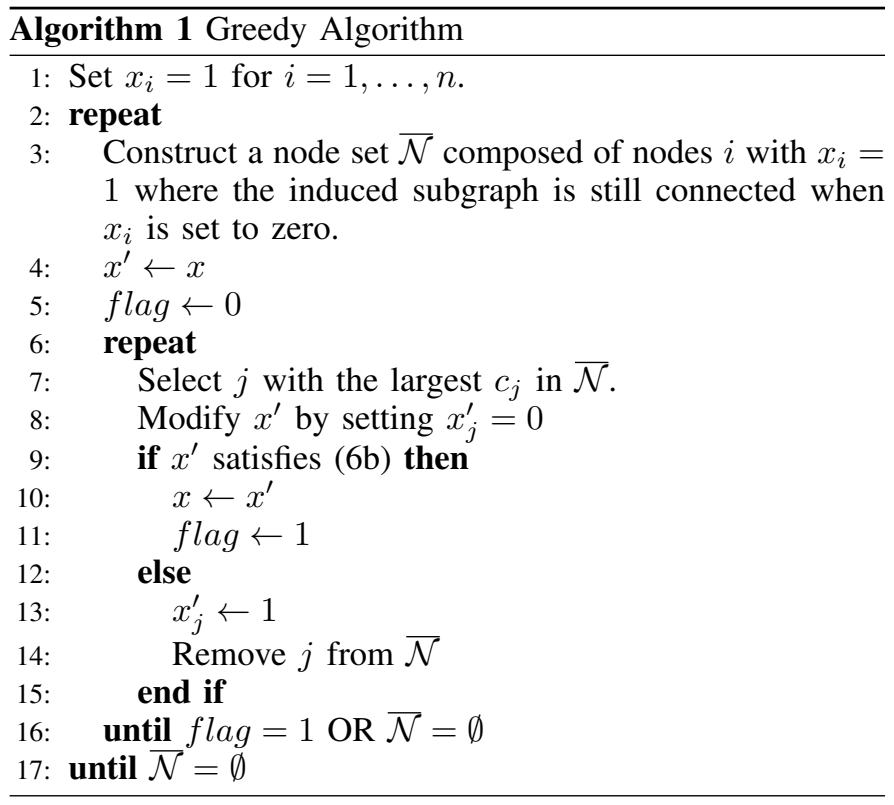

Assume that we have a feasible problem instance. We apply the greedy algorithm to the problem instance to generate a suboptimal solution. Its pseudocode is given in Algorithm 1. In Line 1 , we start with the feasible $x=\left[x_{1}, \ldots, x_{n}\right]=[1, \ldots, 1]$ explained in Lemma 1 and then go through a certain number of iterations (Lines 2-17). In each iteration, we select those nodes in the subgraph induced by $x$ which will not disconnect the subgraph if we remove them from the subgraph and we call this selection $\overline{\mathcal{N}}$ (Line 3). For example, Fig. 2 shows a graph $\hat{G}$ of six nodes, where a dot $i$ and a hole $j$ mean $x_{i}=1$ and $x_{j}=0$, respectively. In this case, we have $\overline{\mathcal{N}}=\{1,3,6\}$. We can see that the resultant $x^{\prime}$ formed by removing any one node in $\overline{\mathcal{N}}$ will still satisfy Constraints (6d)-(6h). Then we attempt to deselect the one (e.g., node $j$ ) with the highest cost 


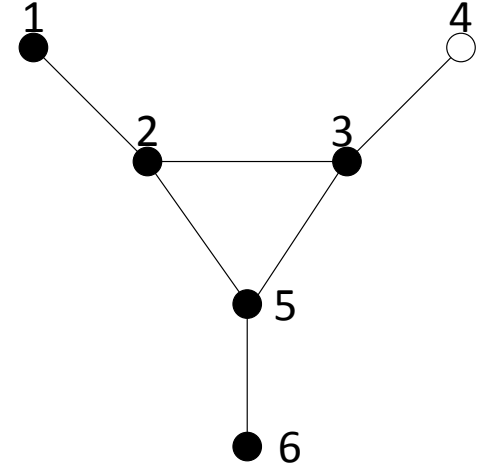

Fig. 2. Node selection in the greedy algorithm.

$c_{j}$ in $\overline{\mathcal{N}}$ (Line 7). If the resultant $x^{\prime}$ satisfies $(6 \mathrm{~b}), x^{\prime}$ is a feasible point and we proceed to the next iteration (Lines 911). Otherwise, we remove $j$ from $\overline{\mathcal{N}}$ (Line 14). Instead of deselecting $j$ (Line 13), we deselect the one with the next highest cost. The iterations terminate when no nodes remain in $\overline{\mathcal{N}}$ (Line 17). The final solution $x$ is the best determined by the greedy algorithm.

\section{Simulation}

As discussed in Section IV, if the demand requirements are all positive, we have two approaches to tackle EVCSPP, i.e., the iterative method for MIP and our proposed greedy algorithm. We perform a series of simulation to evaluate their performance. All simulations are run on the same computer with Intel Core 2 Quad CPU at $2.40 \mathrm{GHz}$ and $4 \mathrm{~GB}$ of RAM, and conducted in the MATLAB environment. For the MIP approach, we adopt the CPLEX solver [12] with YALMIP [13]. Each instance of $G$ is constructed by randomly placing $n$ nodes an area of $100 \times 100 \mathrm{~km}^{2}$, where the cost $c_{i}$ is assigned to a random value in the range of $(0,1]$. For simplicity, we assume that the nodes are interconnected and the length of the shortest path of each pair of nodes is determined by the Euclidean distance between them. As explained in Section III, we can produce $\hat{G}$ from $G$. Then we can check the feasibility of each instance with Corollary 2.

First, we verify the performance of the two approaches with respect to the computation time and the objective function value. We produce 100 random graphs with 10 nodes, where $D, f_{i}$ and $F_{i}$, for all $i$, are set to $80 \mathrm{~km}, 0.5$, and 1 , respectively. The simulation results are given in Table I. The graphs are feasible when $\alpha$ is equal to one. When $\alpha$ decreases, the number of feasible cases resulted will also decrease as we have stronger constraint (6b). The matched cases indicates the number of cases out of the feasible ones producing the same objective function values by the two approaches. The other columns show the average results among the feasible cases. Time ${ }_{\text {MIP }}$ and Time ${ }_{\text {Greedy }}$ indicate the computation times for MIP and the greedy approaches, respectively. Both computation times do not vary much with $\alpha . \mathrm{Obj}_{\mathrm{MIP}}, \mathrm{Obj}_{\mathrm{Greedy}}$, and $\mathrm{Obj}_{\text {upper }}$ represent the objective function values of the solutions obtained from the MIP approach, the greedy algorithm, and the upper bound mentioned in Lemma 1, respectively. In general, the MIP approach produces better solutions than the greedy one but the performance gap is very small (see Table I). The greedy

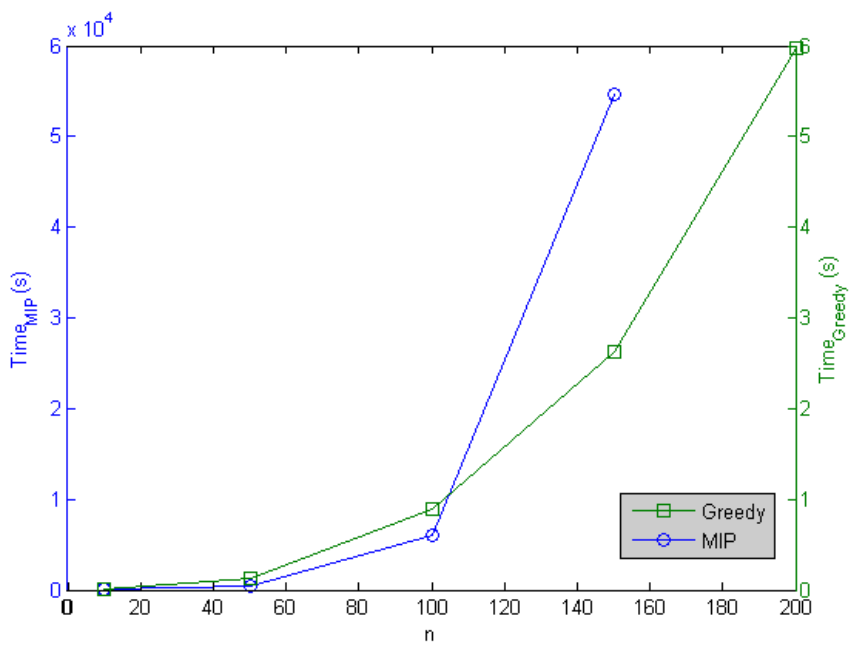

Fig. 3. Computation times for different problem sizes

algorithm can generate the same solutions as the MIP does in many cases. However, the MIP one suffers from much longer computation time.

In the second test, we study how the computation time changes with the problem size. The setting is similar here but we fix $\alpha$ to one for different values of $n$. We generate 100 feasible cases for $n$ equal to 10,50,100,150, and 200. Fig. 3 shows the average computation times for the MIP and greedy approaches. Both the computation times for both increase with $n$ but the one for MIP grows in a much faster fashion. Note that the result of MIP for $n=200$ is not shown because it is not computable by YALMIP/CPLEX due to the out-of-memory problem. This implies the MIP approach is not suitable for large problems.

In the third test, we investigate how the computation time varies with the number of "one" in the solution. Recall that the greedy algorithm always start with the solution of all ones and then it converts some ones to zeros according to the network structure of the problem until no further changes are possible. Hence, the greedy algorithm requires less time when the optimal solution contains more ones. Now we focus on cases with 50 nodes with all $f_{i}=0.5$. Fig. 4 gives the average computation times with different value of $F$, where $F_{i}$ 's for all $i$ are set to $F$. According to (6b), more charging stations should be included in $\mathcal{N}_{i}^{\alpha D}$ with $F$. Hence, the computation time for the greedy algorithm also decreases. However, the computation time for MIP is steady with different values of $F$. The reason is that the greedy algorithm makes use of the network property while the MIP one does not.

To summarize, in terms of solution quality, the MIP approach is better than the greedy one. However, it is only applicable to problems of small dimensions and its computation time is substantially longer than the greedy algorithm. On the other hand, the greedy algorithm is much more efficient and it can produce suboptimal solutions with very good quality.

\section{CONCLUSION}

Gasoline is a heavily demanded natural resource and most is consumed on transportation. Its electrification can relieve our 
TABLE I. SimUlation RESULtS FOR $n=10$ AND $D=80$

\begin{tabular}{c|c|c|c|c|c|c}
\hline \hline$\alpha$ & Matched/Feasible cases & Time $_{\text {MIP }}(\mathrm{s})$ & Time $_{\text {Greedy }}(\mathrm{s})$ & $\mathrm{Obj}_{\mathrm{MIP}}$ & $\mathrm{Obj}_{\text {Greedy }}$ & Obj $_{\text {upper }}$ \\
\hline 1 & $86 / 100$ & 6.4384 & 0.0038 & 0.5579 & 0.5803 & 4.8883 \\
0.9 & $88 / 100$ & 6.3939 & 0.0039 & 0.7100 & 0.7353 & 4.8883 \\
0.8 & $86 / 99$ & 6.4003 & 0.0041 & 1.0263 & 1.0492 & 4.8883 \\
0.7 & $80 / 97$ & 6.2656 & 0.0042 & 1.3207 & 1.3624 & 4.8883 \\
0.6 & $69 / 88$ & 6.1545 & 0.0042 & 1.7492 & 1.7849 & 4.8883 \\
0.5 & $54 / 63$ & 6.1255 & 0.0042 & 2.3549 & 2.3902 & 4.8883 \\
0.4 & $23 / 28$ & 6.0744 & 0.0037 & 3.2389 & 3.2667 & 4.8883 \\
0.3 & $5 / 5$ & 5.7598 & 0.0036 & 3.6983 & 3.6983 & 4.8883 \\
\hline \hline
\end{tabular}

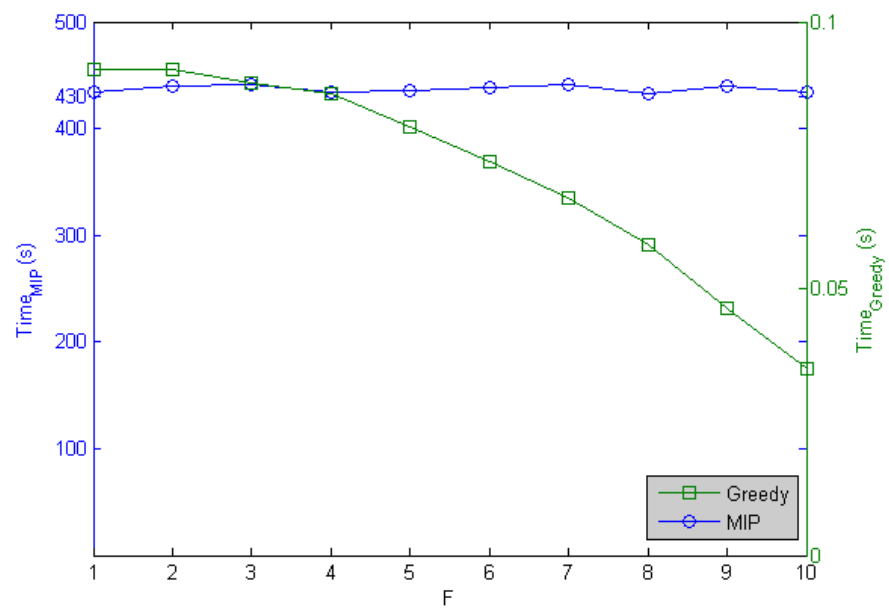

Fig. 4. Computation times for different numbers of ones in the solutions.

dependence on gasoline and tremendously reduce the amount of harmful gases released, which partially constitute global warming and worsen our health. In the $21^{\text {st }}$ century, advancing EV technologies has become one of the keys to boost a nation's economy and maintain (and improve) people's quality of living. For long-term planning, modernizing our cities with EVs is of utmost importance. EVs will be integrated into the transportation system seamlessly and this will help make our cities "smart". To do this, we need to determine the best locations to construct charging stations in the city. In this paper, we focus on human factors rather than technological ones. An EV should always be able to access a charging station within its driving capacity anywhere in the city.

Our contributions in this paper include: 1) formulating the problem, 2) identifying the properties of the problem, and 3) developing an efficient greedy algorithm. We formulate the problem as an optimization model, based on the charging station coverage and the convenience of drivers. We study the NP-hardness of the problem and propose a greedy algorithm based on the network properties of the problem. Simulation results reveal that the greedy algorithm can result in solutions comparable to those obtained from MIP but require much less computation time.

\section{REFERENCES}

[1] S. Chen and L. Tong, "iEMS for large scale charging of electric vehicles: Architecture and optimal online scheduling," in Proceedings of IEEE International Conference on Smart Grid Communications, Tainan City, Taiwan, Nov. 2012.
[2] A. Y. S. Lam, L. Huang, A. Silva, and W. Saad, "A multi-layer market for vechicle-to-grid energy trading in the smart grid," in Proceedings of 1st IEEE INFOCOM Workshop on Green Networking and Smart Grids, Orlando, FL, Mar. 2012.

[3] A. Y. S. Lam, K.-C. Leung, and V. O. K. Li, "Capacity management of vehicle-to-grid system for power regulation services," in Proceedings of IEEE International Conference on Smart Grid Communications, Tainan City, Taiwan, Nov. 2012.

[4] J. J. Q. Yu, V. O. K. Li, and A. Y. S. Lam, "Optimal V2G scheduling of electric vehicles and unit commitment using chemical reaction optimization," in Proceedings of IEEE Congress on Evolutionary Computation, Cancun, Mexico, Jun. 2013.

[5] F. Guo, E. Inoa, W. Choi, and J. Wang, "Study on global optimization and control strategy development for a PHEV charging facility," IEEE Trans. Veh. Technol., vol. 61, pp. 2431-2441, Jul. 2012.

[6] M. Etezadi-Amoli, K. Choma, and J. Stefani, "Rapid-charge electricvehicle stations," IEEE Trans. Power Del., vol. 25, pp. 1883-1887, Jul. 2010.

[7] A. Masoum, S. Deilami, P. Moses, M. Masoum, and A. Abu-Siada, "Smart load management of plug-in electric vehicles in distribution and residential networks with charging stations for peak shaving and loss minimisation considering voltage regulation," IET Generation, Transmission \& Distribution, vol. 5, pp. 877-888, Aug. 2011.

[8] Z. Liu, F. Wen, and G. Ledwich, "Optimal planning of electric-vehicle charging stations in distribution systems," IEEE Trans. Power Del., vol. 28, pp. 102-110, Jan. 2013.

[9] Z. f. Liu, W. Zhang, X. Ji, and L. Ke, "Optimal planning of charging station for electric vehicle based on particle swarm optimization," in Proceedings of IEEE Innovative Smart Grid Technologies - Asia, Tianjin, China, May 2012

[10] S. Wang, R. Crosier, and Y. Chu, "Investigating the power architectures and circuit topologies for megawatt superfast electric vehicle charging stations with enhanced grid support functionality," in Proceedings of IEEE International Electric Vehicle Conference, Greenville, SC, Mar. 2012.

[11] J. M. Conrad, C. P. Gomes, W.-J. van Hoeve, A. Sabharwal, and J. F. Suter, "Wildlife corridors as a connected subgraph problem," Journal of Environmental Economics and Management, vol. 63, pp. 1-18, Jan. 2012.

[12] IBM ILOG CPLEX V12.1 User's Manual for CPLEX, 2009.

[13] J. Löfberg, "YALMIP: A toolbox for modeling and optimization in MATLAB," in Proceedings of IEEE International Symposium on Computer Aided Control Systems Design, Taipei, Taiwan, Sep. 2004. 